\title{
The first detection of radio recombination lines at cosmological distances
}

\author{
K. L. Emig ${ }^{1}$, P. Salas ${ }^{1}$, F. de Gasperin ${ }^{1,2}$, J. B. R. Oonk ${ }^{1,3}$, M. C. Toribio ${ }^{1}$, \\ H. J. A. Röttgering ${ }^{1}$, and A. G. G. M. Tielens ${ }^{1}$ \\ ${ }^{1}$ Leiden Observatory, Leiden University, PO Box 9513, 2300 RA Leiden, The Netherlands \\ e-mail: emig@strw.leidenuniv.nl \\ 2 Hamburger Sternwarte, Universität Hamburg, Gojenbergsweg 112, 21029 Hamburg, Germany \\ 3 ASTRON - The Netherlands Institute for Radio Astronomy, PO Box 2, 7990 AA Dwingeloo, The Netherlands
}

Received 8 August 2018 / Accepted 9 November 2018

\begin{abstract}
Context. Recombination lines involving high principal quantum numbers $(\mathrm{n} \sim 50-1000)$ populate the radio spectrum in large numbers. Low-frequency $(<1 \mathrm{GHz})$ observations of radio recombination lines (RRLs) primarily from carbon and hydrogen offer a new, if not unique, way to probe cold, largely atomic gas and warm, ionised gas in other galaxies. Furthermore, RRLs can be used to determine the physical state of the emitting regions, such as temperature and density. These properties make RRLs, potentially, a powerful tool of extragalactic interstellar medium (ISM) physics. At low radio frequencies, it is conceivable to detect RRLs out to cosmological distances when illuminated by a strong radio continuum. However, they are extremely faint $\left(\tau_{\text {peak }} \sim 10^{-3}-10^{-4}\right)$ and have so far eluded detection outside of the local universe.

Aims. With observations of the radio quasar 3C $190(z=1.1946)$, we aim to demonstrate that the ISM can be explored out to great distances through low-frequency RRLs.

Methods. 3C 190 was observed with the LOw Frequency ARray (LOFAR) and processed using newly developed techniques for spectral analysis.

Results. We report the detection of RRLs in the frequency range $112 \mathrm{MHz}-163 \mathrm{MHz}$ in the spectrum of $3 \mathrm{C} 190$. Stacking $13 \alpha-$ transitions with principal quantum numbers $\mathrm{n}=266-301$, a peak $6 \sigma$ feature of optical depth $\tau_{\text {peak }}=(1.0 \pm 0.2) \times 10^{-3}$ and FWHM $=31.2 \pm 8.3 \mathrm{~km} \mathrm{~s}^{-1}$ was found at $z=1.124$. This corresponds to a velocity offset of $-9965 \mathrm{~km} \mathrm{~s}^{-1}$ with respect to the systemic redshift of $3 \mathrm{C} 190$.

Conclusions. We consider three interpretations of the origin of the RRL emission: an intervening dwarf-like galaxy, an active galactic nucleus (AGN) driven outflow, and the inter-galactic medium. We argue that the recombination lines most likely originate in a dwarflike galaxy $\left(M \sim 10^{9} M_{\odot}\right)$ along the line of sight, although we cannot rule out an AGN-driven outflow. We do find the RRLs to be inconsistent with an inter-galactic medium origin. With this detection, we have opened up a new way to study the physical properties of cool, diffuse gas out to cosmological distances.
\end{abstract}

Key words. galaxies: active - galaxies: ISM - quasars: general - radio lines: galaxies

\section{Introduction}

Under typical conditions found in the interstellar medium (ISM), the recombination of electrons with singly ionised atoms can result in appreciable level populations at high principal quantum numbers (e.g. $\mathrm{n} \sim 300)$. For these Rydberg atoms, the $\alpha$-transitions (i.e. $\Delta \mathrm{n}=1$ ) are so low in energy that they are observable at radio frequencies.

Radio recombination lines (RRLs) that have transitions stimulated by a radiation field have been observed at frequencies $v \lesssim$ $10 \mathrm{GHz}$. The integrated strength of RRLs measured as a function of quantum number, and likewise frequency, is highly dependent upon the physical conditions of the gas. With almost $800 \mathrm{spec}-$ tral lines (per each element) between $10 \mathrm{MHz}$ and $10 \mathrm{GHz}$, this trait makes them powerful tools for understanding the physical properties of the medium, such as temperature, density, thermal pressure, and cloud size.

Within our own Galaxy, stimulated RRLs have been found in regions dominated by either hydrogen or carbon emission. In largely ionised gas with temperatures of $T_{\mathrm{e}} \sim 8000 \mathrm{~K}$ and densities of $n_{\mathrm{e}} \sim 1 \mathrm{~cm}^{-3}$ (e.g. Anantharamaiah 1985), hydrogen RRLs are brightest. This gas phase peaks in intensity at frequencies between $250 \mathrm{MHz}$ and $1000 \mathrm{MHz}$ (Roshi \& Anantharamaiah 2000; Zhao et al. 1996; Shaver et al. 1978; Pedlar et al. 1978). Additionally, cold $\left(T_{\mathrm{e}} \sim 100 \mathrm{~K}\right)$ yet diffuse $\left(n_{\mathrm{e}} \sim 0.05 \mathrm{~cm}^{-3}\right)$ gas can result in carbon being highly stimulated (Shaver 1975). This gas phase occurs in the presence of a radiation field capable of ionising carbon (ionisation potential of $11.3 \mathrm{eV}$ ) yet not hydrogen $(13.6 \mathrm{eV})$. With level populations greatly enhanced by dielectronic capture (Watson et al. 1980), carbon RRLs are the most prominent emitters at $v \lesssim 250 \mathrm{MHz}$ (Konovalenko \& Sodin 1980; Payne et al. 1989; Oonk et al. 2017; Salas et al. 2018).

The detection of low-frequency RRLs is greatly aided towards bright radio sources as the intensity of stimulated transitions is proportional to the strength of the radio continuum, unlike the spontaneous transitions of higher frequency recombination lines associated with HII regions (e.g. Zuckerman \& Ball 1974). This distinction between stimulated and spontaneous transitions is important because it indicates that low-frequency 
RRLs can be studied out to cosmological distances with bright radio sources. The advantages of using stimulated recombination lines to study a variety of regimes was quickly realised by Shaver (1978), including (i) the study of ionised gas in normal galaxies; (ii) the study of the physical conditions in the nuclei of galaxies, quasars and absorption-line systems; (iii) the assessment of the importance of free-free absorption in the spectra of extragalactic radio sources; and (iv) redshift determination for unidentified radio sources.

Out of the 15 extragalactic sources that have been detected via RRLs - all at $v>1 \mathrm{GHz}$ and from nearby galaxies (e.g. for review see Gordon \& Sorochenko 2002; Roy et al. 2008) - only the spectrum of M 82 shows clear evidence of stimulation-dominated emission (Shaver et al. 1978). Non-local sources, such as quasars, were searched at $4.8 \mathrm{GHz}$, but went undetected (Bell et al. 1984). Stifled by instrument capabilities below $\sim 1 \mathrm{GHz}$, few searches in extragalactic sources have been performed and even fewer outside of the local universe (Churchwell \& Shaver 1979). Although RRLs can be very useful probes of the ISM, they are challenging to detect observationally. Their major obstacle stems from the very low peak optical depths of the lines (i.e. with peak fractional absorption of $10^{-3}-10^{-4}$; Gordon \& Sorochenko 2002).

An important step in extragalactic exploration at low frequencies came with the discovery of carbon RRLs at $56 \mathrm{MHz}$ in the nearby starburst galaxy, M 82 (Morabito et al. 2014) using the LOw Frequency ARray (LOFAR; van Haarlem et al. 2013). The advent of sensitive low-frequency telescopes has reinvigorated the field, as the large fractional bandwidth allows for the detection of many RRL transitions simultaneously and stacking can increase the signal-to-noise ratio by an order of magnitude (e.g. Oonk et al. 2015; Salas et al. 2017).

This paper presents the first result of a survey for RRLs at cosmological distances taking advantage of the capabilities afforded by LOFAR with an in-depth study of the $z=1.1946$ radio quasar 3C190 (see Sect. 2). This has resulted in the detection of RRLs outside of the local universe for the first time. While our aim was to investigate cold clouds associated with the HI absorbing gas near to 3C 190, we uncovered something unexpected: RRLs with a large offset in velocity from the radio source.

\section{Target}

We identified 3C 190 as a candidate for RRLs as it is a bright ( $\sim 19 \mathrm{Jy}$ at $140 \mathrm{MHz}$ ), steep-spectrum radio galaxy with $\mathrm{HI}$ detected in absorption (Ishwara-Chandra et al. 2003). It is classified as a reddened quasar (Smith \& Spinrad 1980). Narrow emission lines from [Ne III], [O II], and C III] locate the source at a redshift of $z=1.1946 \pm 0.0005$ (Stockton \& Ridgway 2001). As shown in Fig. 1, the host galaxy of 3C 190 is the central galaxy of a dense environment that is undergoing several major and minor mergers. Optical spectra reveal the presence of an absorption system at $z=1.19565 \pm 0.00004$ observed via $\mathrm{Mg}$ II $\lambda 2798$ and Fe II $\lambda \lambda 2343,2382$ and $\lambda \lambda 2586,2599$ (Stockton \& Ridgway 2001).

Radio observations at $1662 \mathrm{MHz}$ reveal two hot spots spanning 2.6" (Spencer et al. 1991) or $22 \mathrm{kpc}$ in projection. Diffuse emission, stretching to a $4^{\prime \prime}$ extent, indicates the jets may have encountered a dense medium. Using the Giant Metrewave Radio Telescope (GMRT; resolution of $\sim 5^{\prime \prime}$ at $650 \mathrm{MHz}$ ), a broad and complex profile of absorbed HI was detected with five Gaussian components spanning almost $600 \mathrm{~km} \mathrm{~s}^{-1}$ (Ishwara-Chandra et al. 2003). Most of these components are

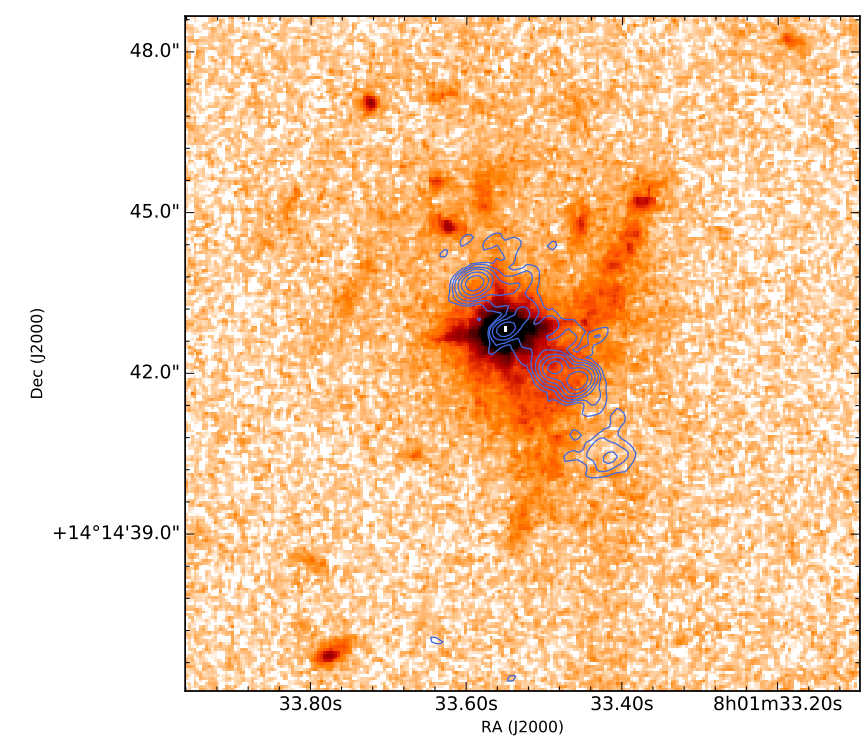

Fig. 1. Quasar 3C 190 (centre) shown in an HST WFPC2 F702W image, where numerous satellite galaxies, a prominent linear feature, and extended diffuse emission can be seen (Stockton \& Ridgway 2001) associated with the system. Superimposed are MERLIN $1658 \mathrm{MHz}$ radio contours at $\sigma \cdot \log _{5}([1,1.5,2,2.5,3,3.5]) \mathrm{mJy} \mathrm{beam}^{-1}$, showing the $22 \mathrm{kpc}$ extent of the lobe hot spots. While 3C 190 is a steep spectrum source, it has a flat spectrum core, and thus we expect low-frequency emission to arise in the radio lobes.

blue-shifted with respect to 3C 190. Of particular interest for carbon RRLs is a deep $\left(\tau_{\text {peak }}=0.0100 \pm 0.0003\right)$ and narrow $\left(\mathrm{FWHM}=66.8 \pm 2.2 \mathrm{~km} \mathrm{~s}^{-1}\right.$ ) feature that lies at a velocity offset of $-210.2 \pm 1 \mathrm{~km} \mathrm{~s}^{-1}$. As the region shows evidence of shocked, turbulent gas, the narrow HI likely results from the radio jet interacting with the ambient medium (Ishwara-Chandra et al. 2003).

\section{Observations and data reduction}

3C 190 was observed with the LOFAR High Band Antennas (HBA) on 14 January 2017 (Project ID LC7_027). Four hours were spent on $3 \mathrm{C} 190$, with ten minutes on the primary calibrator 3C 196 before and after. The 34 stations of the Dutch array were used in HBA_DUAL_INNER mode. Applying the HBAlow analogue filter, we observed between $109.77 \mathrm{MHz}$ and 189.84 MHz. The observing band is split into sub-bands (SBs) of $195.3125 \mathrm{kHz}$ via a poly-phase filter. After which, each SB is divided into 64 channels and recorded at a frequency resolution of $3.0517 \mathrm{kHz}$. While data were taken at $1 \mathrm{~s}$ time intervals, RFI removal and averaging to $2 \mathrm{~s}$ were performed before storing the data.

Processing of the LOFAR data was performed with the SURFSara Grid processing facilities ${ }^{1}$ (e.g. see Mechev et al. 2017,2018 ). While a more detailed description of the data processing can be found in Emig et al. (in prep), we summarise the steps below. Starting with the calibrator data, we flagged the first and last four edge channels of each SB, flagged for RFI using AOflagger (Offringa et al. 2012), selected only the core stations (max baseline $\sim 4 \mathrm{~km}$ ), and averaged the data to resolutions of $6 \mathrm{~s}$ and 32 channels per SB (or $6.1034 \mathrm{kHz}$ channels). Using DPPP (van Diepen \& Dijkema 2018), we solved for diagonal gain. With LoSoTo (de Gasperin et al. 2019) we found the median amplitude solution in time for each channel, creating a

1 https://www.surfsara.nl 


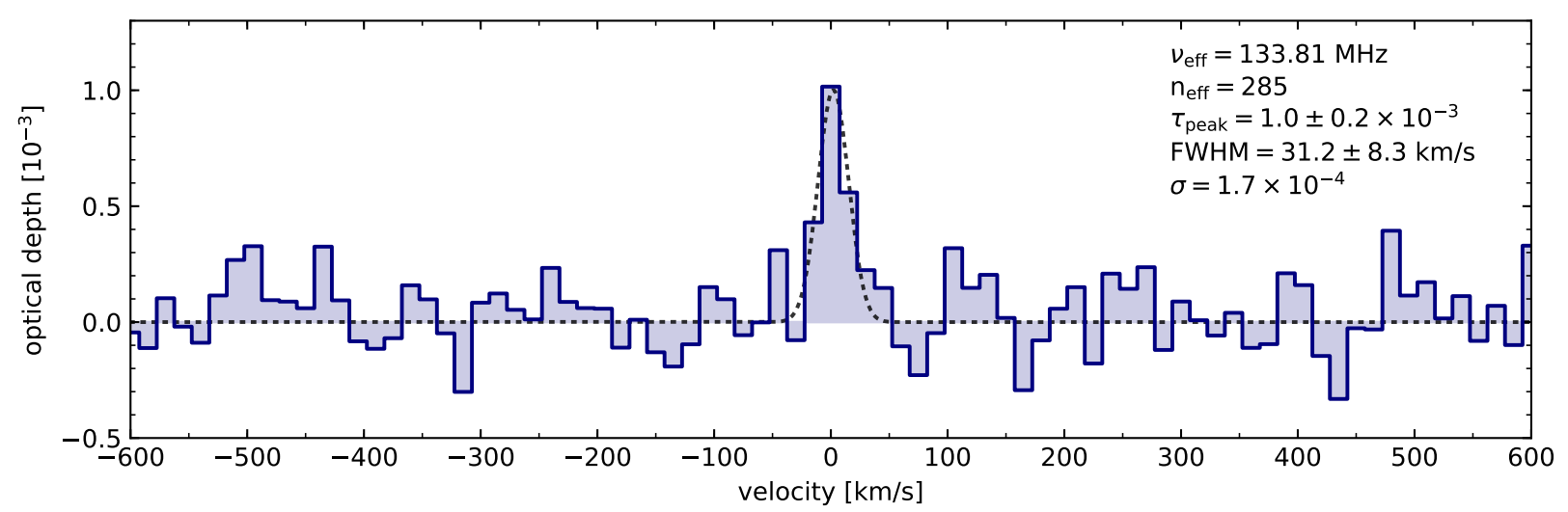

Fig. 2. Detection of a radio recombination line in emission at quantum level $\mathrm{n}_{\mathrm{eff}}=285$, with a velocity centred on $z=1.12405(z=1.12355)$, originating from hydrogen (carbon). This is the average profile of a single line, effectively at $133 \mathrm{MHz}$, as a result of stacking 13 recombination lines in the spectrum of $3 \mathrm{C} 190$.

per channel bandpass solution. Then, a sixth order polynomial across $10 \mathrm{SBs}(2 \mathrm{MHz})$ was fit to take into account the $1 \mathrm{MHz}$ standing wave, the global slope of the bandpass, and smooth over its scatter (e.g. due to the poly phase filter). These effects are expected to be time independent.

Next for the target data, we implemented the same flagging steps as for the calibrator and then applied the bandpass solutions. Flagging once more, for each SB we solved for phase only with DPPP on a $6 \mathrm{~s}$ time interval, using a LOFAR Global Sky Model (van Haarlem et al. 2013) generated sky model of the field. After averaging the data to a $30 \mathrm{~s}$ time resolution, we solved for the amplitude at a resolution of twice per SB. Importing the solution tables of all SBs into LoSoTo, we flagged for outliers (namely to catch RFI due to digital audio broadcasting with broadband effects) and smoothed the solutions in frequency space, with a running Gaussian of 4 SB FWHM, to ensure that any spectral features are not calibrated out. Once we applied the smoothed amplitude solutions, we imaged each channel with WSCLEAN (Offringa et al. 2014), in which multifrequency synthesis was used per SB to make a continuum image and extract the clean components for the channel images (Offringa \& Smirnov 2017).

\section{Spectral stacking}

\subsection{Spectral processing}

In this section we describe the processing of the data post calibration and imaging. In summary, once the channel images were created, we convolved them to the same resolution, extracted flux from a fixed aperture, removed the continuum flux, flagged SB spectra, and stacked the spectra in velocity space.

We first convolved every channel image to the same resolution of 236", a few percent larger than the lowest resolution image, using CASA (McMullin et al. 2007). The flux density was then extracted from a fixed circular aperture of diameter $236^{\prime \prime}$. A spectrum was created for each SB. A fit to the continuum was made to each SB spectrum with a low (1st or 2nd) order polynomial chosen to minimise the chi-square of the fit. For a given redshift, we blanked the channel of the expected frequency of the line when fitting for the continuum. At low frequencies, if stimulated transitions dominate, we have that $I_{\text {line }} \approx I_{\text {cont }} \tau_{\text {line }}$, where the intensity we extract from the observations is $\approx I_{\text {line }}+I_{\text {cont }}$. Therefore, we subtracted the continuum fit and divided by it, which resulted in a measure of the optical depth.
We inspected spectra on a per SB channel basis. In each spectrum we interpolated over channels which had amplitudes higher than five times the spectrum rms. We interpolated over channels for which $\geq 60 \%$ of the visibility data were flagged as well.

Examination of spectra on a per-SB basis was done first by eye to catch clear bandpass-related outliers, for example owing to broadband RFI. We also discarded SBs for which their rms or chi-squared of the continuum fit was a $5 \sigma$ outlier (considering a rolling window of $20 \mathrm{SBs}$ centred on the SB in question). Lastly, if $\geq 40 \%$ (11) of the channels had been clipped or flagged, we did not include the SB in the stack.

In terms of the lines going into the stack, if one of the clipped channels fell within the blanking region of the expected spectral line, the line was not included in the final stack. Furthermore, we required at least three channels on either side of the blanked region to estimate the continuum, otherwise the line was discarded.

Taking the central frequency of each SB, we determined the spectral line closest in frequency and used it to convert the channels to velocity units using the radio definition of velocity, $v^{\text {radio }}=\frac{v_{0}-v}{v_{0}} c$, where $v_{0}$ is rest frequency. At this point, we corrected for the velocity offset necessary for Doppler tracking relative to the local standard of rest (LSR) frame. We then interpolated the velocities to a fixed grid with a channel spacing of $15 \mathrm{~km} \mathrm{~s}^{-1}$.

With the spectra aligned in velocity space, the weighted mean optical depth in each channel was found via

$\left\langle\tau_{\text {chan }}\right\rangle=\frac{\sum_{i=0}^{N}\left(w_{i} \tau_{i}\right)}{\sum_{i=0}^{N} w_{i}}$,

where $i$ represents each SB going into the stack, and the weighting factor was determined by one over the noise variance of each SB spectrum, $w_{i}=\sigma_{i}^{-2}$. The effective frequency and quantum number of the stacked spectrum was determined by the weighted mean values of each SB containing a spectral line.

\subsection{Statistical identification}

We stacked RRLs across redshift space to search for features within the source and along the line of sight. This was sampled at an interval equivalent to the channel width $v=15 \mathrm{~km} \mathrm{~s}^{-1}$ or $\Delta z=10^{-5}$ and ranged over $z=[0,1.31]$. We note that with the flagging procedure above, the number of spectral lines in each stack at a given redshift did not remain constant. While 

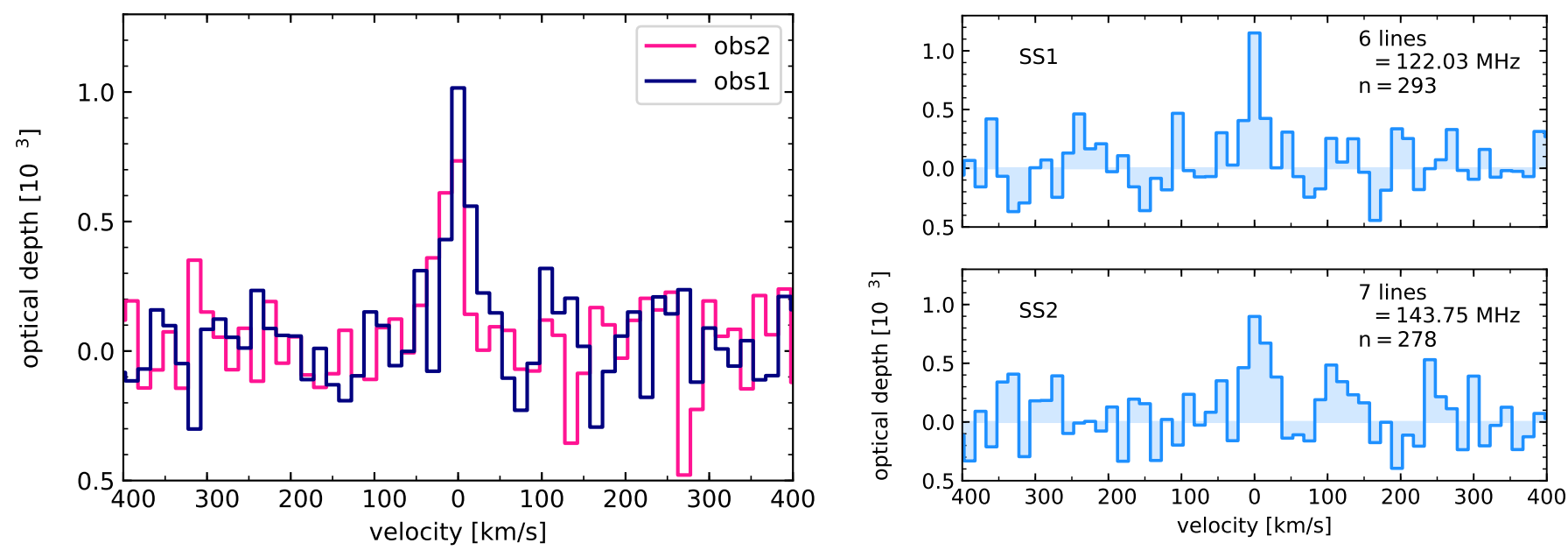

Fig. 3. Left panel: Observation 1 (blue) as in Fig. 2 and the follow-up observation (pink) confirming the feature. These spectra have been Doppler corrected to the LSR. Observation 2, with a factor of 1.6 less time spent on source, is the stack average of 10 lines at quantum number $\mathrm{n}_{\mathrm{eff}}=287$ and $v_{\text {eff }}=130.9 \mathrm{MHz}$. Right panels: we split the spectral lines from Observation 1 into 2 groups, sub-stack 1 (SS1) consisting of the 6 spectral lines with $n>285$ and sub-stack 2 (SS2) containing the 7 lines below.

the following methods are described extensively in Emig et al. (in prep.), we briefly summarise them here.

For each redshift tested, we performed a cross-correlation between a template spectrum and the pre-stacked spectra, both in units of optical depth. The template spectrum was populated with Gaussian line profiles at the location of the spectral lines that contributed to the final stack. The line profiles had a peak of one and their full width at half maximum (FWHM) was set by an assumed blanking region. We then took the cross-correlation and normalised it proportionally with the number of lines that went into the stack, i.e. the total area under the template spectrum. This was the same procedure implemented in Morabito et al. (2014), except we included a normalisation since the number of lines included at each redshift did not remain the same.

As a second test, we took a template spectrum, stacked the spectral lines at an assumed redshift, and integrated the signal within an assumed FWHM. Furthermore, we stacked and integrated the template spectrum at a range of redshifts, from $z-0.01$ to $z+0.01$, at redshift intervals of $10^{-5}$. We then cross-correlated (a) with (b): (a) the integrated optical depth of the template stack as a function of redshift, and $(b)$ the observed integrated optical depth of the stacks at each redshift. With this cross-correlation, we corroborate "mirrors" of the signal that can be found at a $\Delta z=\overline{\Delta v_{\mathrm{n}, \text { eff }}} / v_{\mathrm{n}, \mathrm{eff}}$, or multiples thereof, where $v_{\mathrm{n}, \mathrm{eff}}$ is the frequency of the effective n-level of the stack, and $\overline{\Delta v_{n, \text { eff }}}$ is the average of the change in frequency between $n_{\text {eff }}$ and all other $n$ levels included. We digress to explain the aforementioned mirrors. The distance between each recombination line in frequency space is unique, thus allowing us to accurately determine redshift. However, the difference in spacing between $\alpha$-transitions $\mathrm{n}$ and $\mathrm{n}+1$ is small $(\sim 1 \%)$; in other words, the frequencies at which recombination lines fall are close to being, but not quite, periodic. Therefore mirrors of the feature, which are broadened and reduced in peak intensity compared to the original, occur at offsets in redshift that match the frequency spacing between adjacent lines, or more precisely, $\Delta z=\overline{\Delta v_{\mathrm{n}, \mathrm{eff}}} / \nu_{\mathrm{n}, \mathrm{eff}}$. It was necessary to include the second cross-correlation on account of the low signal-to-noise regime of the lines coupled with poor estimation of the continuum over narrow SBs.

We required that both cross-correlation methods result in a $\geq 5 \sigma$ value at a redshift to report a detection.
Table 1. Spectral properties of the stacked RRLs.

\begin{tabular}{lll}
\hline \hline $\mathrm{n}_{\text {eff }}$ & 285 & \\
$v_{\text {eff }}$ & $133.81 \mathrm{MHz}$ & \\
$\tau_{\text {peak }}$ & $(1.0 \pm 0.2) \times 10^{-3}$ & $19 \pm 4 \mathrm{mJy}^{-1}$ \\
$\mathrm{FWHM}$ & $13.9 \pm 3.7 \mathrm{kHz}$ & $31.2 \pm 8.3 \mathrm{~km} \mathrm{~s}^{-1}$ \\
$\mathrm{rms}$ & $1.7 \times 10^{-4}$ & $3.3 \mathrm{mJy}$ \\
$\int \tau \mathrm{d} v$ & $-14.8 \pm 7.4 \mathrm{~Hz}$ & $640 \pm 320 \mathrm{mJy} \mathrm{km} \mathrm{s}^{-1}$ \\
\hline
\end{tabular}

Notes. The values in the right column were determined by considering a measured flux density of 3C 190 of $19.3 \pm 3.9 \mathrm{Jy}$ at $133.81 \mathrm{MHz}$.

\section{Results}

A significant feature $(\geq 5 \sigma)$ was found in the spectrum of 3C 190 when considering a line blanking of $15 \mathrm{~km} \mathrm{~s}^{-1}$, arising from the $\alpha$-transitions of hydrogen (carbon) at a redshift of $z=$ $1.12405(1.12355) \pm 0.00005$. A redundancy exists between carbon and hydrogen as their $\alpha$-transitions are regularly offset by $149.4 \mathrm{~km} \mathrm{~s}^{-1}$. The stacked feature includes 13 recombination lines of principal quantum numbers $n=266-301$, and it has an effective frequency of $v_{\mathrm{eff}}=133.81 \mathrm{MHz}$ and quantum level of $n_{\text {eff }}=285$. Increasing the blanking region further to $50 \mathrm{~km} \mathrm{~s}^{-1}$, we find the averaged spectral feature (Fig. 2) to have a $31.2 \pm 8.3 \mathrm{~km} \mathrm{~s}^{-1}$ width, thus an under-sampled Gaussian with our velocity resolution, and an average integrated strength per line of $\int \tau \mathrm{d} v=-14.8 \pm 7.4 \mathrm{~Hz}$ at $6.3 \sigma$. Properties of the line and spectrum are listed in Table 1.

The per-channel coverage is about two times lower in the blanked region versus the non-blanked. With the weighting described by Eq. (1), the noise outside of the blanked region is effectively $\sqrt{2}$ times lower than the rms reported in Table 1 . At $z=1.12405$, there are a total of $36 \mathrm{RRLs}$ between $112 \mathrm{MHz}-$ $165 \mathrm{MHz}$. However, 13 were included in the final stack. There were 22 lines that fell within three channels of the SB edge. An additional spectral line was discarded owing to a poor SB bandpass, identified as an outlier in both its rms and continuum fit.

As further validation, we confirm the RRL detection (1) with a second observation, (2) by performing jack-knife tests (Miller 1974), (3) stacking in two independent sub-groups, and (4) stacking other sources in the field at the same redshift. These 
tests all give further confidence to the detection and are described below. Additionally, stacking 3C 190 at other redshifts is consistent with noise.

(1) A second observation of 3C 190 was obtained on 03 May 2017 with the same observational set-up, but with poor ionospheric conditions and thus only $2.5 \mathrm{~h}$ of usable data. Having been taken several months apart, the Doppler correction differs by $32 \mathrm{~km} \mathrm{~s}^{-1}$. As shown on the left side of Fig. 3 in pink, a $4.6 \sigma$ feature, including ten spectral lines at $\mathrm{n}_{\mathrm{eff}}=287$ and $v_{\mathrm{eff}}=130.93 \mathrm{MHz}$, is again seen at $z=1.12405(z=1.12355)$.

(2) Furthermore, jack-knife tests, as in Oonk et al. (2014), were performed on the stacked spectrum, in which we iteratively stack the spectrum, each time discarding one line from the stack. The line properties of the stacks do not differ substantially, indicating that a single SB is not responsible for the signal.

(3) We also split the lines into two groups (see also Oonk et al. 2014) resulting in two independent stacks, shown in the right-hand side of Fig. 3. Sub-stack 1 (SS1) consists of six spectral lines with $v_{\text {eff }}=122.03 \mathrm{MHz}$ and $\mathrm{n}_{\mathrm{eff}}=293$; Substack 2 (SS2) consists of seven lines with $v_{\text {eff }}=143.75 \mathrm{MHz}$ and $n_{\text {eff }}=278$. The line profiles are consistent among the two stacks. The line is more narrow but higher in its peak optical depth in SS1 as compared with SS2, which is consistent with Doppler broadening effects.

(4) Lastly, we stack the next two brightest sources in the field, 3C 191 and $4 C+15.22$, at $z=1.12405$ as well. While their noise is significantly higher and thus so are their limits on the optical depth, we confirm no prominent feature is seen in their spectra. This gives further confidence that the detection is not an instrumental systematic.

\section{RRL Modelling and Interpretation}

RRLs allow us to make constraints on the emitting gas properties inferred from the observables of central velocity, line width, and line integrated strength. We discuss the constraints they place on the origin of the detected line. The difference in redshift between 3C 190 and the RRL-emitter implies a velocity difference of $-9965 \mathrm{~km} \mathrm{~s}^{-1}$, corresponding to a (luminosity) distance from 3C 190 of $81 \mathrm{Mpc}$.

To characterise the emitting gas, we use models of radio recombination line emission. Ideally, with multiple RRL detections distributed in frequency, models can be fit to derive gas properties. However, with only one data point, we instead explore a range of input physical conditions (density and temperature) and obtain a range in physical properties (emission measure, column density, path length, and pressure). We use these physical properties to constrain different, possible interpretations on the origin of the emitting gas.

The integrated optical depth of stimulated recombination lines at low frequencies is described by

$$
\int \tau \mathrm{d} v=2.046 \times 10^{6} \mathrm{~Hz} \cdot \exp \left(\chi_{\mathrm{n}}\right)\left(\frac{T_{\mathrm{e}}}{\mathrm{K}}\right)^{-5 / 2} \frac{E M}{\mathrm{~cm}^{-6} \mathrm{pc}} b_{\mathrm{n}} \beta_{\mathrm{n}}
$$

for $\alpha$ transitions (e.g. Shaver 1975; Salgado et al. 2017a). Here $\chi_{\mathrm{n}}=1.58 \times 10^{5} \mathrm{~K} /\left(\mathrm{n}^{2} T_{\mathrm{e}}\right), \mathrm{n}$ is the quantum level, and $E M$ is the emission measure expressed as $E M=n_{\mathrm{e}} n_{\text {ion }} L_{\text {ion }}$ for electron density $n_{\mathrm{e}}$, ion density $n_{\text {ion }}$, and path length $L_{\text {ion }}$. The coefficients that describe the gas departure from local thermodynamic equilibrium, $b_{\mathrm{n}} \beta_{\mathrm{n}}$, require detailed modelling of the atomic physics,
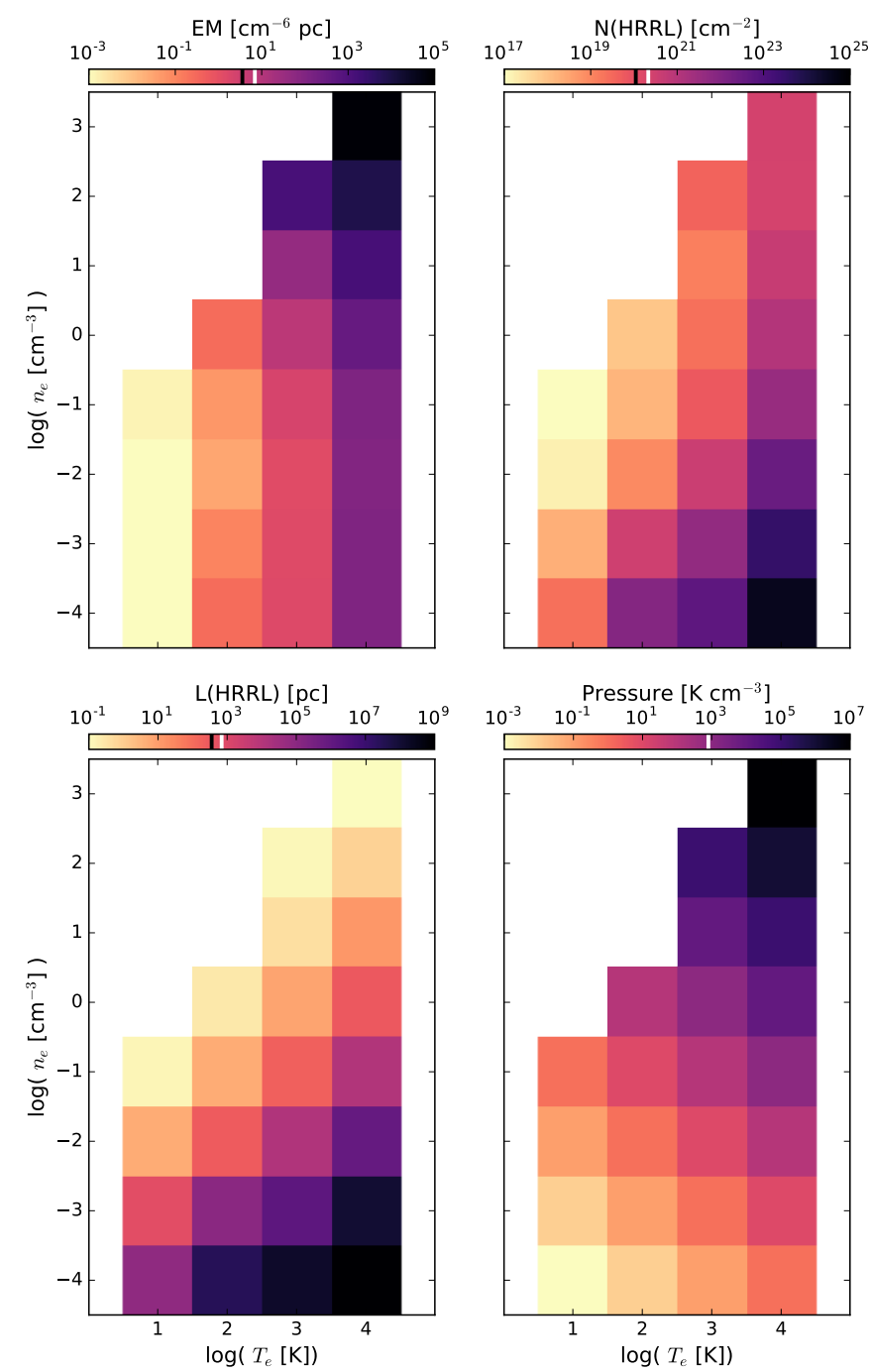

Fig. 4. Derived physical properties from modelling of hydrogen RRL departure coefficients. For input physical conditions, electron density, and temperature, shown on the axes, we computed a departure coefficient $\left(b_{\mathrm{n}} \beta_{\mathrm{n}}\right)$ via RRL modelling. Plugging in the $b_{\mathrm{n}} \beta_{\mathrm{n}}$, the integrated optical depth, quantum number, $n_{\mathrm{e}}$ and $T_{\mathrm{e}}$ into Eq. (2), we derive the emission measure (top left panel) and corresponding column density (top right panel) and path length (bottom left panel) of the RRL emission. Additionally we show the electron pressure (bottom right panel). Values representing the warm, ionised phase of a face-on (black) and an edge-on (white) Milky Way are shown with dashes in the colour bars. Hydrogen RRLs are expected to be most prominent from warm, $T \sim 1000-10000 \mathrm{~K}$, gas.

for which we refer to the comprehensive low-frequency models of Salgado et al. (2017a,b).

We explored models with electron densities ranging from $10^{-4} \mathrm{~cm}^{-3}-10^{3} \mathrm{~cm}^{-3}$, electron temperatures of $10 \mathrm{~K}-10^{4} \mathrm{~K}$, and with four different radiation fields. We wanted to cover a wide range of parameters that include the typical temperatures of carbon (10 K-100 K; Oonk et al. 2017; Salas et al. 2017) and of hydrogen $\left(10^{3} \mathrm{~K}-10^{4} \mathrm{~K}\right.$; Anantharamaiah 1985; Heiles et al. 1996) RRL emitting gas. We note, the assumptions made for dielectronic capture in Salgado et al. (2017a) are relevant only for $T<15000 \mathrm{~K}$ (Watson et al. 1980). The densities were chosen to cover typical parameters found within our Galaxy in diffuse clouds, HII region outskirts, and extended low-density phases of the ISM (e.g. Tielens 2005; Ferriere 2001). Moreover, we incorporated more extreme conditions which resemble those 

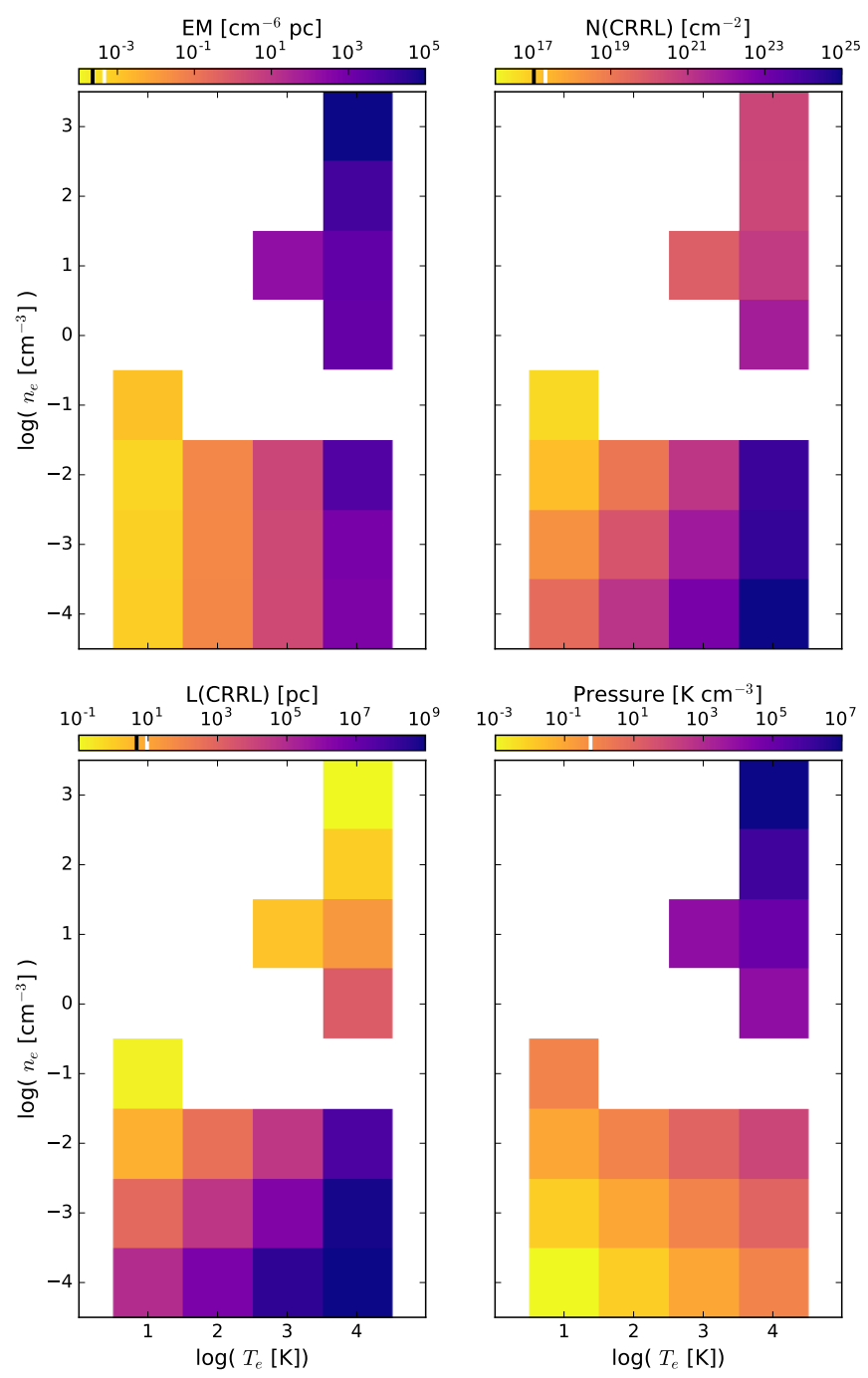

Fig. 5. Same physical properties as in Fig. 4, except for carbon RRL modelling of the departure coefficients and the Milky Way values are representative of its cold, neutral medium. Carbon RRLs are expected to be most prominent from cold, $T \sim 10-100 \mathrm{~K}$, gas.

of the (less dense) inter-galactic medium (IGM) and (possibly, more dense) AGN environments. The radiation fields we consider have (1) only cosmic microwave background (CMB) radiation (for $z=0$ ), (2) CMB radiation and a synchrotron field with $\beta=-2.6$ (e.g. de Oliveira-Costa et al. 2008; Klein et al. 2018) and scaled to $T_{\mathrm{r}}=800 \mathrm{~K}$ at $100 \mathrm{MHz},(3)$ to $T_{\mathrm{r}}=2000 \mathrm{~K}$, and (4) to $T_{\mathrm{r}}=10^{5} \mathrm{~K}$. For the $19.3 \pm 3.9 \mathrm{Jy}$ flux density of $3 \mathrm{C} 190$ at $133.81 \mathrm{MHz}$, the synchrotron brightness temperature is given by $T_{\mathrm{b}}=(1+z) 4.7 \times 10^{4} \mathrm{~K} \mathrm{sr}^{-1}$. For an absorber $81 \mathrm{Mpc}$ away with an estimated $\Omega \sim 2 \times 10^{-7} \mathrm{sr}$, the effective radiation temperature from 3C 190 is negligible $\left(T_{\mathrm{r}}=0.02 \mathrm{~K}\right)$. This implies that if the RRLs originate in an intervening absorber, the continuum from $3 \mathrm{C} 190$ does not contribute significantly to $T_{\mathrm{r}}$. On the other hand, material that is $\sim 15 \mathrm{kpc}$ away (or at least within a $\mathrm{Mpc}$ ) would see a significantly higher radiation temperature, $T_{\mathrm{r}} \sim 10^{5} \mathrm{~K}$.

Starting from Eq. (2), we can solve for $E M$ by inputting observed quantities (i.e. $\int \tau \mathrm{d} v=-14.8 \pm 7.4 \mathrm{~Hz}$ at $\mathrm{n}_{\mathrm{eff}}=285$ ) and the computed departure coefficient and assumed temperature. The physical properties obtained are shown in Figs. 4 and 5 for hydrogen and carbon, respectively. We assume all ionised electrons originate from their RRL element, and ionised frac- tions of $X_{\mathrm{e}}=1$ and $X_{\mathrm{e}}=A_{\mathrm{C}}=1.4 \times 10^{-4}$ (Sofia et al. 2004) in the respective cases. The figures show values obtained for $T_{\mathrm{r}}=2000 \mathrm{~K}$; these are representative of the radiation fields considered. The properties derived for the three coolest radiation fields do not differ by more than $\sim 20 \%$. For the radiation field of $T_{\mathrm{r}}=10^{5} \mathrm{~K}$, the results are qualitatively similar; they can be found in Fig. A.1. We indicate values that the Milky Way would be observed to have if placed at $z=1.124$; we assume cold gas extends to $R=25 \mathrm{kpc}$ (Dickey et al. 2009) with parameters of the warm, ionised medium, and cold, neutral medium found in Tielens (2005). We also assume that the background continuum is emitted from a region $4^{\prime \prime}$ in extent, equivalent to $33.3 \mathrm{kpc}$ at the redshift of 3C 190.

We consider five scenarios to explain the RRLs: (i) hydrogen or (ii) carbon RRLs from an intervening galaxy, (iii) hydrogen or (iv) carbon RRLs from the AGN outflow, and (v) hydrogen RRLs from the IGM. It is not immediately clear whether the RRL originates from hydrogen or carbon because of two main issues. Firstly, a regular separation of $149.4 \mathrm{~km} \mathrm{~s}^{-1}$ exists for the Bohr-like $\alpha$-transitions between the two elements. Secondly, at the frequency at which we detected the feature, rest-frame $284 \mathrm{MHz}$, cold (carbon origin) and warm (hydrogen origin) gas phases have both been observed in RRLs within our own Galaxy (e.g. Anantharamaiah 1985). In the following sections, we give a brief description of each scenario and its RRL modelling constraints.

\subsection{Intervening, dwarf-like galaxy}

The RRLs would originate from the disc or in extended material of a dwarf-like galaxy along the line of sight. The galaxy should be small in mass and size such that it is not visible in Hubble Space Telescope (HST) imaging shown in Fig. $1\left(M_{\mathrm{U}}>27.2\right)$. The narrow line-width of the RRL indicates a face-on orientation consistent with cool phases of the ISM for small galaxies, whether they be from the disc or from a halo component (Tumlinson et al. 2013). In this object, star formation would be possible and thus reasonable for a stimulated radiation field. Since we do not find a counterpart for the RRL in existing spectroscopic observations of the quasar (Stockton \& Ridgway 2001), the RRL source should overlap with only the radio emission and not the optical. Furthermore, the high redshift and small mass suggests that the galaxy would be low in metallicity.

\subsubsection{Hydrogen RRLs in an intervening galaxy}

In scenario (i), the RRL would originate from hydrogen and thus largely ionised gas, either in the disc or in extended material of a dwarf-type galaxy.

We find sensible results (see Fig. 4) for models with temperatures $10^{2}<T_{\mathrm{e}} / \mathrm{K}<10^{5}$ and densities $10^{-2}<n_{\mathrm{e}} / \mathrm{cm}^{-3}<10$, in agreement with RRLs observed in the disc of the Milky Way (Anantharamaiah 1985; Heiles et al. 1996). The model results for plausible interpretations of the RRL-emitting gas are listed in Table 2. We list the model input and derived physical properties for models with $T_{\mathrm{r}}=800 \mathrm{~K}$. Also listed is an estimate of the mass of warm ionised material; we assume a galaxy of $R=5.5 \mathrm{kpc}$ and thus a surface filling factor of $\phi=0.1$. We calculate the mass for a face-on disc as $M=m_{\mathrm{H}} n_{\mathrm{H}} \pi R^{2} h$, where $h$ is the scale height of the phase, $h=L / \phi$, and $L$ is the path length derived from the model.

We also make note of warm, ionised gas that could originate in the halo or circum-galactic medium of a small galaxy. The densities would be slightly lower, closer to $n_{\mathrm{e}} \leq 10^{-2} \mathrm{~cm}^{-3}$ 
Table 2. Model results for which only plausible interpretations are listed.

\begin{tabular}{|c|c|c|c|c|c|c|c|c|c|c|c|}
\hline Scenario & $\begin{array}{c}n_{\mathrm{e}} \\
\left(\mathrm{cm}^{-3}\right) \\
\end{array}$ & $\begin{array}{c}T_{\mathrm{e}} \\
(\mathrm{K}) \\
\end{array}$ & $\begin{array}{c}P / k \\
\left(\mathrm{~K} \mathrm{~cm}^{-3}\right) \\
\end{array}$ & $b_{\mathrm{n}} \beta_{\mathrm{n}}$ & $\begin{array}{c}E M \\
\left(\mathrm{~cm}^{-6} \mathrm{pc}\right)\end{array}$ & $\begin{array}{c}N(R R L) \\
\left(\mathrm{cm}^{-2}\right)\end{array}$ & $\begin{array}{c}L \\
(\mathrm{pc})\end{array}$ & $\phi$ & $\begin{array}{c}M \\
\left(M_{\odot}\right) \\
\end{array}$ & $\begin{array}{c}Q \\
\left(\text { phot s }^{-1}\right)\end{array}$ & $\begin{array}{c}\mathrm{SFR} \\
\left(M_{\odot} \mathrm{yr}^{-1}\right)\end{array}$ \\
\hline \multicolumn{12}{|c|}{ (i) Galaxy, hydrogen } \\
\hline & 1 & $10^{3}$ & $10^{3}$ & -28.9 & 7.8 & $2 \times 10^{19}$ & 7.8 & 0.10 & $2 \times 10^{8}$ & $3.6 \times 10^{53}$ & 3.9 \\
\hline & 1 & $10^{4}$ & $10^{4}$ & -205 & 350 & $1 \times 10^{21}$ & 350 & 0.10 & $8 \times 10^{9}$ & $2.5 \times 10^{54}$ & 27 \\
\hline & 0.1 & $10^{3}$ & $10^{2}$ & -103 & 2.2 & $7 \times 10^{19}$ & 220 & 0.10 & $5 \times 10^{8}$ & $1.0 \times 10^{53}$ & 1.1 \\
\hline & 0.1 & $10^{4}$ & $10^{3}$ & -587 & 120 & $4 \times 10^{21}$ & 12000 & 0.66 & $* 3 \times 10^{10}$ & $1.9 \times 10^{54}$ & 21 \\
\hline & 0.01 & $10^{3}$ & 10 & -159 & 1.4 & $4 \times 10^{20}$ & 14000 & 0.73 & $* 3 \times 10^{9}$ & $1.4 \times 10^{53}$ & 1.5 \\
\hline \multicolumn{12}{|c|}{ (ii) Galaxy, carbon } \\
\hline & $\begin{array}{l}0.1 \\
0.01\end{array}$ & $\begin{array}{l}10 \\
10\end{array}$ & $10^{2.9}$ & $\begin{array}{l}-1.31 \\
-3.37\end{array}$ & $\begin{array}{c}0.001 \\
0.0006\end{array}$ & $\begin{array}{l}4 \times 10^{17} \\
2 \times 10^{17}\end{array}$ & $\begin{array}{l}0.14 \\
5.5\end{array}$ & $\begin{array}{l}0.10 \\
0.10\end{array}$ & $9 \times 10^{9}$ & & $\begin{array}{l}\sim 0.1 \\
\star \sim 1\end{array}$ \\
\hline \multicolumn{12}{|c|}{ (iii) Outflow, hydrogen } \\
\hline & 10 & $10^{3}$ & $10^{4}$ & -5.23 & 44 & $1 \times 10^{19}$ & 0.43 & 0.0007 & $* 9 \times 10^{7}$ & & \\
\hline \multicolumn{12}{|c|}{ (iv) Outflow, carbon } \\
\hline & 0.1 & 10 & $10^{3.9}$ & -0.76 & 0.002 & $8 \times 10^{16}$ & 0.25 & 0.0005 & $* 4 \times 10^{9}$ & & \\
\hline
\end{tabular}

Notes. $n_{\mathrm{e}}, T_{\mathrm{e}}$ : input electron density and electron temperature. For scenario (i) and (ii), a radiation temperature of $T_{\mathrm{r}}=800 \mathrm{~K}$ was considered, and for scenario (iii) $T_{\mathrm{r}}=10^{5} \mathrm{~K}$ was considered. $P$ : thermal pressure of the input temperature and density, where $P / k \sim\left(n_{\mathrm{e}} / X_{\mathrm{e}}\right) T_{\mathrm{e}} . b_{\mathrm{n}} \beta_{\mathrm{n}}$ : departure coefficient derived from the models. $E M$ : RRL emission measure derived from the model output and Eq. (2). $N, L$ : RRL column density and path length corresponding to the $E M . \phi$ : surface filling factor of the object in the beam. $M$ : total mass of a disk-like, face-on galaxy of $R=5.5 \mathrm{kpc}$. *assumes a spherical cloud in calculating the mass. $Q$ : ionization rate needed to maintain ionized gas of the input $n_{\mathrm{e}}, T_{\mathrm{e}}$. SFR: star-formation rate determined from $Q .{ }^{\star}$ denotes the SFR was inferred from the cold neutral medium mass $M$.

(Tumlinson et al. 2017), and the path lengths longer, as the gas is distributed outside of the disc. Possibilities for this are also listed in Table 2, except we consider this material to be spherically distributed for the mass estimate. For spherical geometry, we let $R^{3}=3 / 4 L \cdot(16.6 \mathrm{kpc})^{2}$ to calculate the implied true radius of the clump, and extract the beam filing factor.

It is a relevant exercise to place an upper limit on the temperature of the gas assuming (non-)thermal motions Dopplerbroaden the line width. As defined in Brocklehurst \& Seaton (1972), a Doppler-broadened line with FWHM $(\Delta v)$ is given by $\Delta v=30.25 \mathrm{~km} \mathrm{~s}^{-1}\left(\frac{m_{\mathrm{p}}}{m} \frac{T}{2 \times 10^{4} \mathrm{~K}}\right)^{1 / 2}$, where $m_{\mathrm{p}}$ is the proton mass, $m$ is the nuclear mass, and in this work $T$ we take as the electron temperature. We find an upper limit for hydrogen gas of $T_{\mathrm{e}}=21300 \mathrm{~K}$.

As we find a broad range of physical conditions $\left(10^{2}<\right.$ $T_{\mathrm{e}} / \mathrm{K}<10^{4.3}$ and $\left.10^{-2}<n_{\mathrm{e}} / \mathrm{cm}^{-3}<10\right)$ that could be interpreted as hydrogen RRLs in an intervening, dwarf galaxy, we estimate the number of ionising photons needed to maintain this gas phase. As described in Rubin (1968), the ionisation rate, $Q$, needed to maintain the implied physical conditions is given by $Q$ [photons s ${ }^{-1}$ ] $=4.1 \times 10^{-10} n_{\mathrm{e}} n_{\text {ion }} V T_{\mathrm{e}}^{-0.8}$ in a total volume $V$. The computed value of each plausible interpretation is shown in Table 2. Moreover, we calculate star formation rates (SFRs) from these ionisation rates, via SFR $\left[M_{\odot} \mathrm{yr}^{-1}\right]=Q \cdot 1.08 \times 10^{-53}$ (Kennicutt 1998).

\subsubsection{Carbon RRLs in an intervening galaxy}

In scenario (ii), RRLs from carbon would arise in cold, diffuse gas clouds within the mid-plane of a galaxy. With conservative constraints on thermal pressure, $P<10^{5} \mathrm{~K} \mathrm{~cm}^{-3}$ (Jenkins \& Tripp 2001; Herrera-Camus et al. 2017), and on the column density, $N(\mathrm{CRRL})<10^{18} \mathrm{~cm}^{-2}$ such that $N(\mathrm{HI})<$ $10^{22} \mathrm{~cm}^{-2}$ for a carbon abundance of $A_{\mathrm{C}}=1.4 \times 10^{-4}$, we rule out many of the possibilities considered in Fig. 5. However, we find two plausible sets of physical conditions for this scenario, placing limits on electron density of $10^{-3}<n_{\mathrm{e}} / \mathrm{cm}^{-3}<1$ and electron temperature of $T_{\mathrm{e}}<100 \mathrm{~K}$. We list the results in Table 2, along with an estimate of the mass of cold neutral material as described above for the face-on disc, letting $n_{\mathrm{H}}=n_{\mathrm{e}} / A_{\mathrm{C}}$.

Since this is a viable scenario, we derive integrated SFRs from the mass estimate of the cold, atomic gas. Referencing the relation Lopez-Sanchez et al. (2018) have found for local volume dwarf and spiral galaxies that have gas fractions dominated by HI, we find the estimated masses of $2 \times 10^{9} M_{\odot}$ and $9 \times 10^{9} M_{\odot}$ to have SFRs of $\sim 0.1 M_{\odot} \mathrm{yr}^{-1}$ and $1 M_{\odot} \mathrm{yr}^{-1}$, respectively.

\subsection{AGN-driven outflow}

In this scenario, RRLs would originate in gas outflowing as a result of the quasar or of the jet impacting the medium, for instance the optical linear feature apparent in Fig. 1.

There are notable reasons why this interpretation is less likely. In this system, nothing in the present literature indicates gas is moving at velocities higher than $\sim 600 \mathrm{~km} \mathrm{~s}^{-1}$ (Stockton \& Ridgway 2001), one to two orders of magnitude below that of the RRL velocity. Furthermore, the narrow line width of the RRL is hard to maintain with a very high velocity.

Despite these indications, we investigated the scenario based on the following reasons. Jet interactions causing cold and ionised gas to outflow up to $\sim 1000 \mathrm{~km} \mathrm{~s}^{-1}$ have been observed in a number of AGN (e.g. Morganti et al. 2005). Via Sloan Digital Sky Survey (SDSS) optical spectra of $\sim 17000$ quasars, the majority of associated absorbers are found out to $2000 \mathrm{~km} \mathrm{~s}^{-1}$ $4000 \mathrm{~km} \mathrm{~s}^{-1}$, with tails to $10000 \mathrm{~km} \mathrm{~s}^{-1}$, albeit for warmer gas (Chen \& Pan 2017). Additionally, warm gas with narrow line widths and velocities of $-14050 \mathrm{~km} \mathrm{~s}^{-1}$ has been observed in radiation-driven outflows (Hamann et al. 2011), and with velocities up to $-3000 \mathrm{~km} \mathrm{~s}^{-1}$ in low-ionisation species, $900 \mathrm{pc}$ away from the quasar (Xu et al. 2018). 
In the case of 3C 190, its jet speed is estimated to be $0.22 \mathrm{c}$, even out to its current $\sim 15 \mathrm{kpc}$ scale. This is based on orientation (Best et al. 1995) and electron ageing (Katz-Stone \& Rudnick 1997). The jet indeed appears to be interacting with the optical linear feature seen through the diffuse radio emission surrounding the hot spots (Katz-Stone \& Rudnick 1997) as well as displaced [O II] and a resulting cavity-like feature (Stockton \& Ridgway 2001). Again, we point out that the displaced [O II] has a FWHM of $85 \mathrm{~km} \mathrm{~s}^{-1}$ and reaches velocities of $-600 \mathrm{~km} \mathrm{~s}^{-1}$ relative to $3 \mathrm{C} 190$.

Two possible outflow scenarios could be (1) that the RRL originates in ablated [OII] material and is carried to high velocities, approaching that of the jet or wind speeds, and (2) that the RRL cools out of the shock heated material, and as it approaches $T \sim 10^{4} \mathrm{~K}$, it condenses.

For a $500 \mathrm{pc}$ cloud with a velocity dispersion of $31 \mathrm{~km} \mathrm{~s}^{-1}$, the dissipation time of the cloud is $10^{7} \mathrm{yrs}$. With a bulk motion of $10000 \mathrm{~km} \mathrm{~s}^{-1}$, this gas could reach $\sim 100 \mathrm{kpc}$ before dissipating. This implies that observing this type of gas cloud is conceivable.

\subsubsection{Hydrogen RRLs in an AGN-driven outflow}

In this scenario (iii), hydrogen RRLs would originate in (partially) ionised gas. With the higher densities we explore, collisional broadening may cause significant line broadening. We adopt the following expression for the Lorentzian FWHM due to collisions with electrons for $\alpha$-transitions (Salgado et al. 2017b),

$\Delta v_{\mathrm{col}} \approx \frac{n_{\mathrm{e}}}{\mathrm{cm}^{-3}}\left(\frac{10^{a} \mathrm{n}^{\gamma_{\mathrm{col}}}}{\pi}\right) \mathrm{Hz}$

where $a$ and $\gamma_{\mathrm{col}}$ depend on the gas temperature (values for which be found in Salgado et al. 2017b) and $n$ is the quantum level. We use this prescription to place an upper limit on the density of $n_{\mathrm{e}}<15 \mathrm{~cm}^{-3}$ for our range of temperatures. For a density of $n_{\mathrm{e}}=10 \mathrm{~cm}^{-3}$ and temperatures of $T_{\mathrm{e}}=10^{3} \mathrm{~K}-$ $10^{4} \mathrm{~K}$, the thermal pressure would be elevated compared to typical ISM values (see Fig. 4). High pressures have been found in various gas phases of outflowing material (e.g. Santoro et al. 2018; Oosterloo et al. 2017; Holt et al. 2011). We list physical conditions that would indeed be possible within this scenario in Table 2.

\subsubsection{Carbon RRLs in an AGN-driven outflow}

In scenario (iv), RRLs would originate in an outflow, but from material colder and more dense than the previous scenario. As we stated in the intervening galaxy example, we expect $N(\mathrm{CRRL})<10^{18} \mathrm{~cm}^{-2}$, since the corresponding neutral hydrogen column density limit is $10^{22} \mathrm{~cm}^{-2}$. We note that for gas with $T_{\mathrm{e}}<100 \mathrm{~K}$ and density of $0.01<n_{\mathrm{e}} / \mathrm{cm}^{-3}<1$, the path length derived from the model is $L=0.25 \mathrm{pc}$. Assuming that the gas is a sphere, this path length would imply a radius of $370 \mathrm{pc}$ and hence a surface filling factor of $5 \times 10^{-4}$. These and other physical properties listed in Table 2 do not seem unreasonable. We conclude that this scenario could explain the RRLs we observe.

\subsection{Hydrogen RRLs from the intervening IGM}

Lastly, we consider gas with typical properties of an isolated IGM cloud at $z \sim 1$, with temperature $T_{\mathrm{e}} \sim 10^{4.5} \mathrm{~K}$ and densities $n_{\mathrm{e}}<10^{-4} \mathrm{~cm}^{-3}$ (McQuinn 2016). To test this scenario (v), we extended the RRL models down to densities of $10^{-4} \mathrm{~cm}^{-3}$, but notice that at low densities and high temperatures, we find unreasonably large path lengths, approaching $10^{5}-10^{7} \mathrm{kpc}$, in order to match the observed feature (see Fig. 4). Ultimately, we determine it is unfeasible to reproduce the observed integrated optical depth of the RRL with an IGM cloud.

\section{Conclusions}

Using LOFAR, we have identified RRLs centred at $133.8 \mathrm{MHz}$ in the spectrum of 3C 190, as a result of stacking $13 \alpha$-transitions at $z=1.124$ (Fig. 2). This is the first detection of RRLs outside of the local universe.

At low frequencies, recombination lines can occur in diffuse gas from stimulated transitions in hydrogen and carbon. Since their transitions are regularly spaced $\sim 150 \mathrm{~km} \mathrm{~s}^{-1}$ apart, an ambiguity exists in determining the species of origin. However, carbon and hydrogen RRLs originate in distinctly different types of gas. Carbon arises in cold, neutral gas clouds, and hydrogen RRLs arise in warm, largely ionised material.

In this paper, we demonstrate how RRLs can be used to study the physical properties in these types of gas clouds. We model the non-LTE effects responsible for strong stimulation; inputting physical conditions (temperature and density), we constrain the origin of the gas (Table 2). We find the RRL could be explained by hydrogen $\left(10^{2}<T_{\mathrm{e}} / \mathrm{K}<10^{4.3}\right.$ and $\left.10^{-2}<n_{\mathrm{e}} / \mathrm{cm}^{-3}<10\right)$ or carbon $\left(T_{\mathrm{e}}<10^{2} \mathrm{~K}\right.$ and $\left.10^{-3}<n_{\mathrm{e}} / \mathrm{cm}^{-3}<1\right)$ emission in an intervening, dwarf galaxy $\left(M \sim 10^{9} M_{\odot}\right)$, roughly $80 \mathrm{Mpc}$ from 3C 190. Although we consider it to be less likely, we cannot rule out the possibility that the RRL emitter is outflowing from the radio-loud quasar at $\sim 10000 \mathrm{~km} \mathrm{~s}^{-1}$, from either hydrogen or carbon emission. Lastly, we rule out an IGM origin.

Since the RRLs in the spectrum of 3C 190 have no counterpart in existing observations, follow-up investigations are crucial. Observations targeting RRLs at a higher and a lower frequency give the best indication of success; they would distinguish between the carbon and hydrogen RRL origin and further constrain the physical conditions of the gas. An intervening galaxy seen only against the radio emission may reveal itself, slightly offset from the quasar, as an absorber in integral-fieldspectroscopy observations taken with high-spatial resolution. If the RRL originates from cold, neutral gas, HI $21 \mathrm{~cm}$ absorption may be found at the redshifted frequency. HI has been searched for over only a small velocity interval centred on the systemic velocity and gas at $\sim 10000 \mathrm{~km} \mathrm{~s}^{-1}$ would have been missed.

The detection of RRLs in the spectrum of 3C 190 has opened up a new way to study the physical properties of the ISM out to cosmological distances. The LOFAR Two Metre Sky Survey (Shimwell et al. 2017, 2019) of the Northern Hemisphere is uniquely suited to characterise RRL emitters in a large population of sources.

Acknowledgements. The authors would like to thank the referee for constructive and positive feedback. The authors would like to thank Alan Stockton, Anita Richards, and Ralph Spencer for their help in acquiring supplementary data. We also thank Aayush Saxena, Madusha Gunawardhana, Rogier Windhorst, Turgay Culgar, and Nastasha Wijers for useful discussions. KLE, PS, JBRO, HJAR, and AGGMT acknowledge financial support from the Netherlands Organization for Scientific Research (NWO) through TOP grant 614.001.351. AGGMT acknowledges support through the Spinoza premier of the NWO. MCT acknowledges financial support from the NWO through funding of Allegro. FdG is supported by the VENI research programme with project number 639.041.542, which is financed by the NWO. Part of this work was carried out on the Dutch national e-infrastructure with the support of the SURF Cooperative through grant e-infra $160022 \& 160152$. This paper is based (in part) on results obtained with International LOFAR Telescope (ILT) equipment under project code LC7_027. LOFAR (van Haarlem et al. 2013) is the 
K. L. Emig et al.: Radio recombination lines at cosmological distances

LOw Frequency ARray designed and constructed by ASTRON. It has observing, data processing, and data storage facilities in several countries, which are owned by various parties (each with their own funding sources) and are collectively operated by the ILT foundation under a joint scientific policy. The ILT resources have benefitted from the following recent major funding sources: CNRS-INSU, Observatoire de Paris and Universite d'Orleans, France; BMBF, MIWF-NRW, MPG, Germany; Science Foundation Ireland (SFI), Department of Business, Enterprise and Innovation (DBEI), Ireland; NWO, The Netherlands; The Science and Technology Facilities Council, UK; and the Ministry of Science and Higher Education, Poland. KLE would like to welcome Zada Gray Emig Tibbits into this world. Software: APLpy (Robitaille \& Bressert 2012), astropy (Astropy Collaboration 2018), CASA (McMullin et al. 2007), CRRLpy (Salas et al. 2016), DPPP (van Diepen \& Dijkema 2018), iPython (Perez \& Ganger 2007), LoSoTo (de Gasperin et al. 2019), matplotlib (Hunter 2007), WSCLEAN (Offringa et al. 2014)

\section{References}

Anantharamaiah, K. R. 1985, JApA, 6, 203

Astropy Collaboration (Price-Whelan, A. M., et al.) 2018, AJ, 156, 123

Bell, M. B., Seaquist, E. R., Mebold, U., Reif, K., \& Shaver, P. 1984, A\&A, 130, 1

Best, P. N., Bailer, D. M., Longair, M. S., \& Riley, J. M. 1995, MNRAS, 275, 1171

Brocklehurst, M., \& Seaton, M. J. 1972, MNRAS, 157, 179

Chen, Z.-F., \& Pan, D.-S. 2017, ApJ, 848, 79

Churchwell, E., \& Shaver, P. A. 1979, A\&A, 77, 316

de Gasperin, F., Dijkema, T. J., Drabent, A., et al. 2019, A\&A, 622, A5 (LOFAR SI)

de Oliveira-Costa, A., Tegmark, M., Gaensler, B. M., et al. 2008, MNRAS, 388 247

Dickey, J. M., Strasser, S., Gaensler, B. M., et al. 2009, ApJ, 693, 1250

Ferriere, K. M. 2001, Rev. Mod. Phys., 73, 1031

Gordon, M. A., \& Sorochenko, R. L. 2002, Radio Recombination Lines, Their Physics and Astronomical Applications (Dordrecht: Kluwer Academic Publishers)

Hamann, F., Kanekar, N., Prochaska, J. X., et al. 2011, MNRAS, 410, 1957

Heiles, C., Reach, W. T., \& Koo, B.-C. 1996, ApJ, 466, 191

Herrera-Camus, R., Bolatto, A., Wolfire, M., et al. 2017, ApJ, 835, 201

Holt, J., Tadhunter, C. N., Morganti, R., \& Emonts, B. H. C. 2011, MNRAS, 410, 1527

Hunter, J. D. 2007, Comput. Sci. Eng., 9, 90

Ishwara-Chandra, C. H., Dwarakanath, K. S., \& Anantharamaiah, K. R. 2003, JApA, 24, 37

Jenkins, E. B., \& Tripp, T. M. 2001, ApJS, 137, 297

Katz-Stone, D. M., \& Rudnick, L. 1997, ApJ, 479, 258

Kennicutt, R. C. J. 1998, ARA\&A, 36, 189

Klein, U., Lisenfeld, U., \& Verley, S. 2018, A\&A, 611, 55

Konovalenko, A. A., \& Sodin, L. G. 1980, Nature, 283, 360

Lopez-Sanchez, A. R., Lagos, C. D. P., Young, T., \& Jerjen, H. 2018, MNRAS, 480, 210

McMullin, J. P., Waters, B., Schiebel, D., Young, W., \& Golap, K. 2007, Astronomical Data Analysis Software and Systems XVI, ASP Conf. Ser., 376, 127
McQuinn, M. 2016, ARA\&A, 54, 313

Mechev, A. P., Oonk, J. B. R., Danezi, A., et al. 2017, in Proc. Int. Symp. Grids Clouds 2017, Taipei

Mechev, A. P., Plaat, A., Oonk, J. B. R., Intema, H. T., \& Röttgering, H. J. A. 2018, Astron. Comput., 2, 117

Miller, R. G. 1974, Biometrika, 61, 1

Morabito, L. K., Oonk, J. B. R., Salgado, F., et al. 2014, ApJ, 795, L33

Morganti, R., Tadhunter, C. N., \& Oosterloo, T. A. 2005, A\&A, 444, L9

Offringa, A. R., \& Smirnov, O. 2017, MNRAS, 471, 301

Offringa, A. R., van de Gronde, J. J., \& Roerdink, J. B. T. M. 2012, A\&A, 539, 95

Offringa, A. R., McKinley, B., Hurley-Walker, N., et al. 2014, MNRAS, 444, 606

Oonk, J. B. R., van Weeren, R. J., Salgado, F., et al. 2014, MNRAS, 437, 3506

Oonk, J. B. R., Morabito, L. K., Salgado, F., et al. 2015, in Proc. Adv. Astrophysics with the Square Kilometre Array 2014, 139

Oonk, J. B. R., van Weeren, R. J., Salas, P., et al. 2017, MNRAS, 465, 1066

Oosterloo, T., Oonk, J. B. R., Morganti, R., et al. 2017, A\&A, 608, A38

Payne, H. E., Anantharamaiah, K. R., \& Erickson, W. C. 1989, ApJ, 341, 890

Pedlar, A., Davies, R. D., Hart, L., \& Shaver, P. A. 1978, MNRAS, 182, 473

Perez, F., \& Ganger, B. E. 2007, Comput. Sci. Eng., 9, 21

Robitaille, T., \& Bressert, E. 2012, Astrophysics Source Code Library, [record ascl: 1208.017]

Roshi, D. A., \& Anantharamaiah, K. R. 2000, ApJ, 535, 231

Roy, A. L., Goss, W. M., \& Anantharamaiah, K. R. 2008, A\&A, 483, 79

Rubin, R. H. 1968, ApJ, 154, 391

Salas, P., Morabito, L., Salgado, F., Oonk, J. B. R., \& Tielens, A. G. G.M. 2016, CRRLpy: First Pre-release

Salas, P., Oonk, J. B. R., van Weeren, R. J., et al. 2017, MNRAS, 467, 2274

Salas, P., Oonk, J. B. R., van Weeren, R. J., et al. 2018, MNRAS, 2511, 2496

Salgado, F., Morabito, L. K., Oonk, J. B. R., et al. 2017a, ApJ, 837, 141

Salgado, F., Morabito, L. K., Oonk, J. B. R., et al. 2017b, ApJ, 837, 142

Santoro, F., Rose, M., Morganti, R., et al. 2018, A\&A, 617, A139

Shaver, P. A. 1975, A\&A, 43, 465

Shaver, P. A. 1978, A\&A, 68, 97

Shaver, P. A., Churchwell, E., \& Walmsley, C. M. 1978, A\&A, 64, 1

Shimwell, T. W., Röttgering, H. J. A., Best, P. N., et al. 2017, A\&A, 598, 102

Shimwell, T. W., Tasse, C., Hardcastle, M. J., et al. 2019, A\&A, 622, A1 (LOFAR SI)

Smith, H. E., \& Spinrad, H. 1980, ApJ, 236, 419

Sofia, U. J., Lauroesch, J. T., Meyer, D. M., \& Cartledge, S. I. B. 2004, ApJ, 605, 272

Spencer, R. E., Schilizzi, R. T., Fanti, C., et al. 1991, MNRAS, 250, 225

Stockton, A., \& Ridgway, S. E. 2001, ApJ, 554, 1012

Tielens, A. G. G. M. 2005, The Physics and Chemistry of the Interstellar Medium (Cambridge: Cambridge University Press), 495

Tumlinson, J., Thom, C., Werk, J. K., et al. 2013, ApJ, 777, 59

Tumlinson, J., Peeples, M. S., \& Werk, J. K. 2017, ARA\&A, 55, 389

van Diepen, G., \& Dijkema, T. J. 2018, Astrophysics Source Code Library, [record ascl: 1804.003]

van Haarlem, M. P., Wise, M. W., Gunst, A. W., et al. 2013, A\&A, 556, A2

Watson, W. D., Western, L. R., \& Christensen, R. B. 1980, ApJ, 240, 956

Xu, X., Arav, N., Miller, T., \& Benn, C. 2018, ApJ, 858, 39

Zhao, J.-H., Anantharamaiah, K. R., Goss, W. M., \& Viallefond, F. 1996, ApJ, 472,54

Zuckerman, B., \& Ball, J. A. 1974, ApJ, 190, 35 


\section{Appendix A: RRL Modelling for high radiation temperatures}
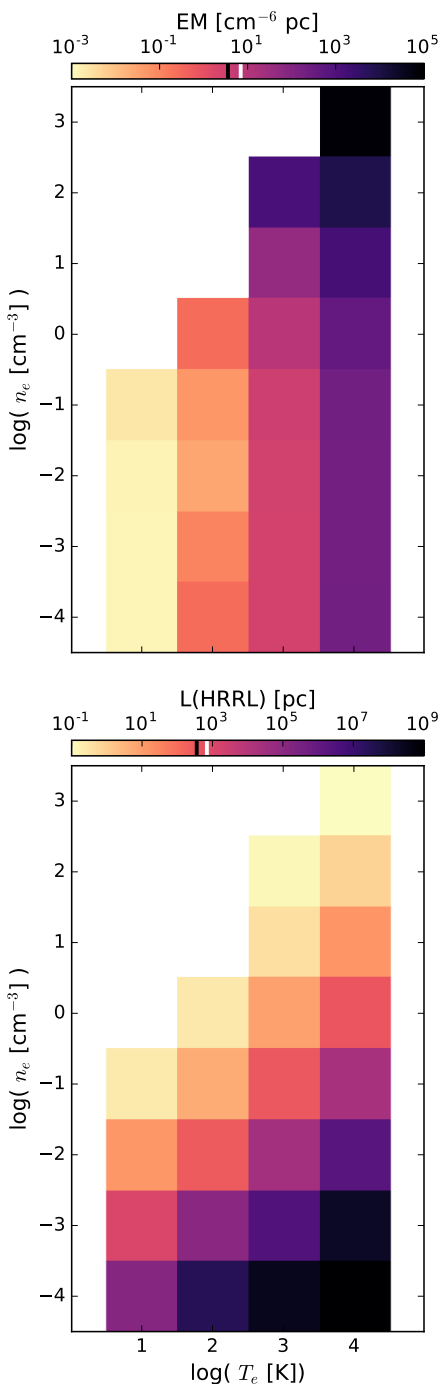
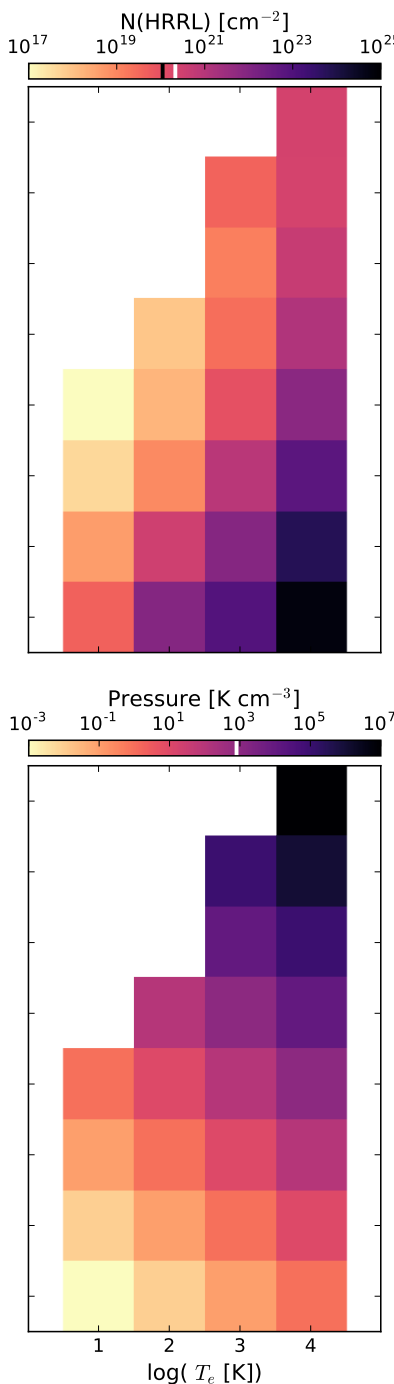
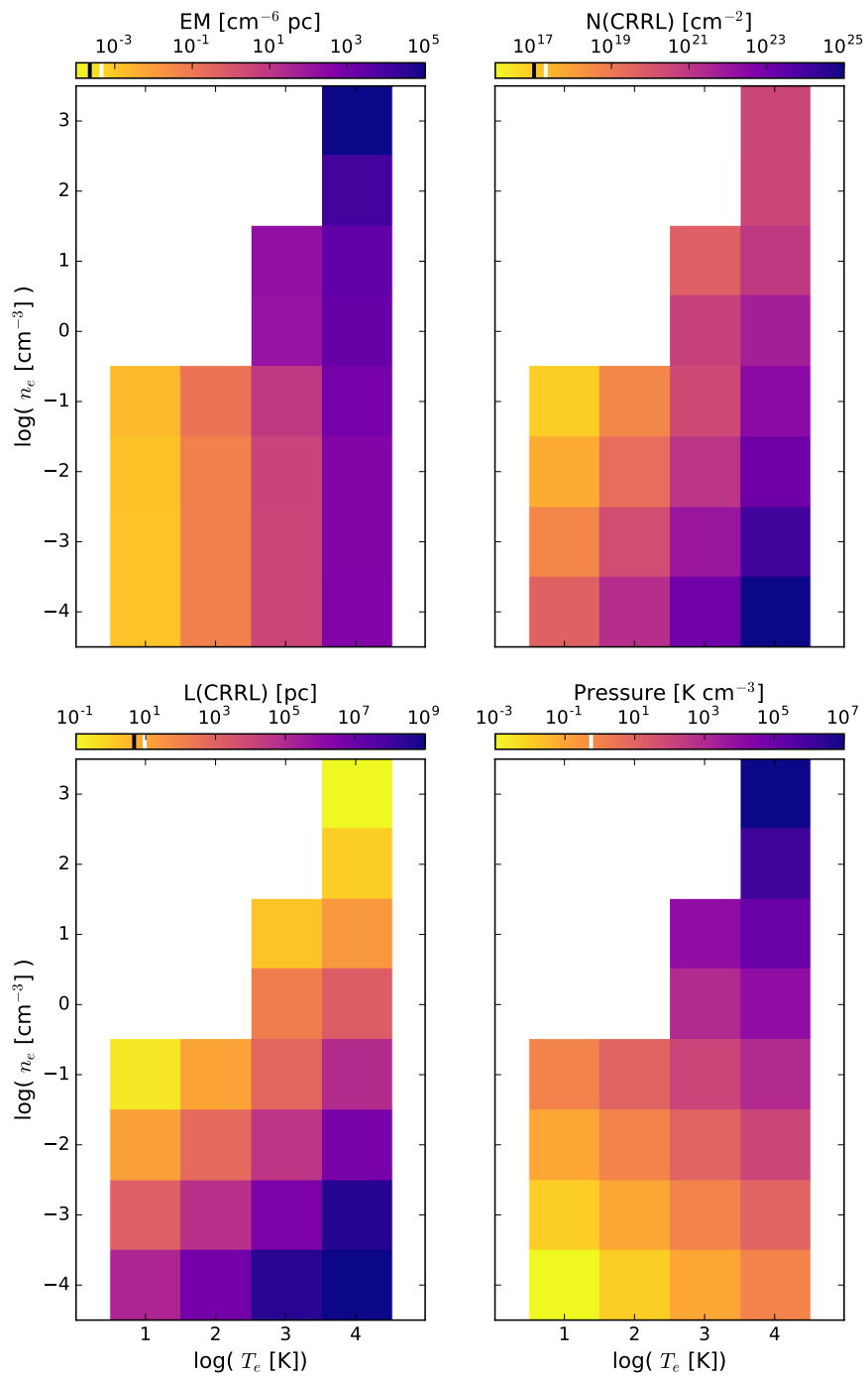

Fig. A.1. Same physical properties as in Fig. 4 and 5, except these results for hydrogen (left panels) and carbon (right panels) have been derived with $T_{\mathrm{r}}=10^{5} \mathrm{~K}$. 